\title{
Guide de présentation des rapports sur les éclosions
}

\author{
Correspondance : ccdr-rmtc@phac-aspc.gc.ca
}

Les rapports sur les éclosions décrivent une éclosion une fois qu'elle est terminée. Ils résument la façon dont l'éclosion a été détectée, les enquêtes qui ont été menées, les interventions qui ont été réalisées pour la contrôler et elles fournissent l'épidémiologie descriptive et les résultats. Ils sont utiles pour déterminer les risques émergents et pour décrire les nouvelles techniques d'enquêtes ou décrire les techniques d'intervention. En général, les rapports sur les éclosions ont une longueur de 2000 à 2500 mots - excluant le résumé, les tableaux et les références.

Le Relevé des maladies transmissibles au Canada (RMTC) a adapté les lignes directrices en matière de rapports du système ORION (Outbreak Reports and Intervention studies Of Nosocomial infection) (1). Étant donné que ces lignes directrices ont été élaborées principalement pour les études d'intervention dans le domaine des infections nosocomiales, elles ont été adaptées aux éclosions communautaires.

Le Tableau 1 présente une liste de vérification des rapports sur les éclosions et la Figure 1 illustre un exemple de courbe épidémique, ou un histogramme dans lequel le nombre de nouveaux cas d'une maladie est calculé par rapport à un intervalle de temps pour décrire une éclosion précise.

Comme dans le cas de toutes les soumissions, vérifier les Renseignements à l'intention des auteurs (publiés en général en janvier chaque année avec le premier numéro de chaque nouveau volume) du Relevé des maladies transmissibles au Canada (RMTC) pour les exigences générales relatives à la préparation et à la soumission des manuscrits.

Tableau 1 : Liste de vérification des éléments à inclure au moment de la déclaration d'une éclosion

\begin{tabular}{|c|c|c|}
\hline $\begin{array}{l}\text { Élément de la } \\
\text { déclaration }\end{array}$ & $\mathbf{N}^{01}$ & Description \\
\hline \multicolumn{3}{|l|}{ Titre } \\
\hline Titre & 1 & $\begin{array}{l}\text { Donner un titre qui comprend le terme « éclosion », la maladie, la population, ou le } \\
\text { lieu et le moment. }\end{array}$ \\
\hline \multicolumn{3}{|l|}{ Résumé } \\
\hline $\begin{array}{l}\text { Résumé } \\
\text { structuré }\end{array}$ & 2 & $\begin{array}{l}\text { Utiliser un format structuré pour le résumé avec les en-têtes de section suivants: } \\
\text { Contexte, Objectifs, Méthodologie, Résultats et Conclusion. }\end{array}$ \\
\hline \multicolumn{3}{|l|}{ Introduction } \\
\hline Milieu & 3 & Décrire le milieu (collectivité, hôpital, etc.) où l'éclosion est survenue. \\
\hline Identification & 4 & Décrire les événements qui ont mené à la découverte du cas index de l'éclosion. \\
\hline Contexte & 5 & $\begin{array}{l}\text { Fournir le contexte scientifique (p. ex. décrire l'organisme et déterminer s'il est } \\
\text { émergent, épidémique, endémique, etc.). }\end{array}$ \\
\hline Justification & 6 & $\begin{array}{l}\text { Déterminer la justification clinique et de santé publique de la déclaration d'une } \\
\text { éclosion (p. ex. la nécessité d'une prise de conscience accrue, la démonstration } \\
\text { d'une nouvelle technique d'enquête ou d'intervention). }\end{array}$ \\
\hline Objectif & 7 & $\begin{array}{l}\text { Exprimer l'objectif du rapport sur l'éclosion. (p. ex. : " L'objectif du présent rapport } \\
\text { est de décrire l'enquête épidémiologique, diagnostique et génétique de l'éclosion qui } \\
\text { a eu lieu en -). }\end{array}$ \\
\hline \multicolumn{3}{|l|}{ Méthodologie } \\
\hline Aperçu & 8 & Déterminer les dates de début et de fin de l'éclosion et la date à laquelle les \\
\hline
\end{tabular}




\begin{tabular}{|c|c|c|}
\hline & & $\begin{array}{l}\text { enquêtes ont commencé. Décrire la façon dont la date de fin a été déterminée, y } \\
\text { compris la période d'incubation et la date du dernier cas déclaré. }\end{array}$ \\
\hline \multirow{2}{*}{$\begin{array}{l}\text { Recherches de } \\
\text { cas et collecte de } \\
\text { données }\end{array}$} & 9 & $\begin{array}{l}\text { Fournir les définitions de cas (y compris les cas confirmés, probables et soumis à } \\
\text { une enquête, le cas échéant). }\end{array}$ \\
\hline & 10 & $\begin{array}{l}\text { Décrire les activités de collecte de données (pour la personne, le moment et le lieu), } \\
\text { y compris l'élaboration d'un questionnaire (antécédents cliniques, évaluation des } \\
\text { facteurs de risque). }\end{array}$ \\
\hline Enquêtes & 11 & $\begin{array}{l}\text { Fournir une description systématique de la façon dont l'éclosion a été étudiée, y } \\
\text { compris } \\
\text { - } \quad \text { les enquêtes en laboratoire; } \\
\text { - } \quad \text { l'échantillonnage environnemental. }\end{array}$ \\
\hline \multirow{3}{*}{$\begin{array}{l}\text { Analyses } \\
\text { épidémiologiques } \\
\text { et statistiques }\end{array}$} & 12 & $\begin{array}{l}\text { Décrire toutes les méthodes d'analyse utilisées pour évaluer l'éclosion (p. ex. } \\
\text { l'analyse des facteurs de risque, l'analyse de survie, l'estimation des taux de } \\
\text { référence). }\end{array}$ \\
\hline & 13 & $\begin{array}{l}\text { Citer les méthodes d'analyse complexes utilisées (p. ex. l'analyse des réseaux } \\
\text { sociaux, l'estimation du taux de reproduction de base). }\end{array}$ \\
\hline & 14 & $\begin{array}{l}\text { Inclure des analyses des sous-groupes, les mesures prises pour contrôler les } \\
\text { interactions et les facteurs de confusion, ainsi que les méthodes utilisées pour } \\
\text { s'occuper des données manquantes et des retards de déclaration. }\end{array}$ \\
\hline Interventions & 15 & $\begin{array}{l}\text { Décrire les mesures cliniques et de santé publique qui ont été mises en place pour } \\
\text { contrôler l'éclosion, y compris, le cas échéant: } \\
\text { - les antécédents d'exposition; } \\
\text { - } \quad \text { l'évaluation des risques pour la santé; } \\
\text { - } \quad \text { les traitements cliniques; } \\
\text { - } \quad \text { les mesures de santé publique (p. ex. la mise en quarantaine, la recherche de } \\
\text { sujets-contacts, la surveillance, les cliniques de vaccination, la communication } \\
\text { des risques, etc.). }\end{array}$ \\
\hline \multicolumn{3}{|l|}{ Résultats } \\
\hline $\begin{array}{l}\text { Épidémiologie } \\
\text { descriptive }\end{array}$ & 16 & Donner un aperçu de ce qui s'est passé pour chaque personne, moment et lieu. \\
\hline $\begin{array}{l}\text { Analyses } \\
\text { accessoires }\end{array}$ & 17 & $\begin{array}{l}\text { Fournir des analyses des sous-groupes et décrire l'évaluation des interactions et } \\
\text { des facteurs de confusion, tel qu'il est indiqué. }\end{array}$ \\
\hline Complications & 18 & Déterminer les complications, par exemple les hospitalisations et les décès. \\
\hline $\begin{array}{l}\text { Courbe } \\
\text { épidémique }\end{array}$ & 19 & $\begin{array}{l}\text { Fournir une figure montrant la courbe épidémique. Dans le titre, inclure la maladie, } \\
\text { la population, et le lieu et le moment (année). }\end{array}$ \\
\hline $\begin{array}{l}\text { Tableau de la } \\
\text { fréquence }\end{array}$ & 20 & $\begin{array}{l}\text { Inclure un tableau présentant les caractéristiques démographiques (p. ex. âge et } \\
\text { sexe) et la fréquence des symptômes, s'il y a lieu. }\end{array}$ \\
\hline \multicolumn{3}{|l|}{ Discussion } \\
\hline $\begin{array}{l}\text { Principaux } \\
\text { résultats }\end{array}$ & 21 & $\begin{array}{l}\text { Résumer les principales constatations liées à l'objectif du rapport, en mettant } \\
\text { l'accent sur les aspects nouveaux ou importants de l'éclosion et sur leur importance. }\end{array}$ \\
\hline Comparaison & 22 & Tenir compte de ces résultats par rapport à la documentation actuelle. \\
\hline $\begin{array}{l}\text { Forces et } \\
\text { faiblesses }\end{array}$ & 23 & Cerner les points forts et les points faibles de l'enquête sur l'éclosion. \\
\hline Conclusion & 24 & S'assurer que les conclusions abordent l'objectif et font le suivi des résultats. \\
\hline
\end{tabular}


Figure 1 : Exemple de courbe épidémique pour une éclosion de rougeole

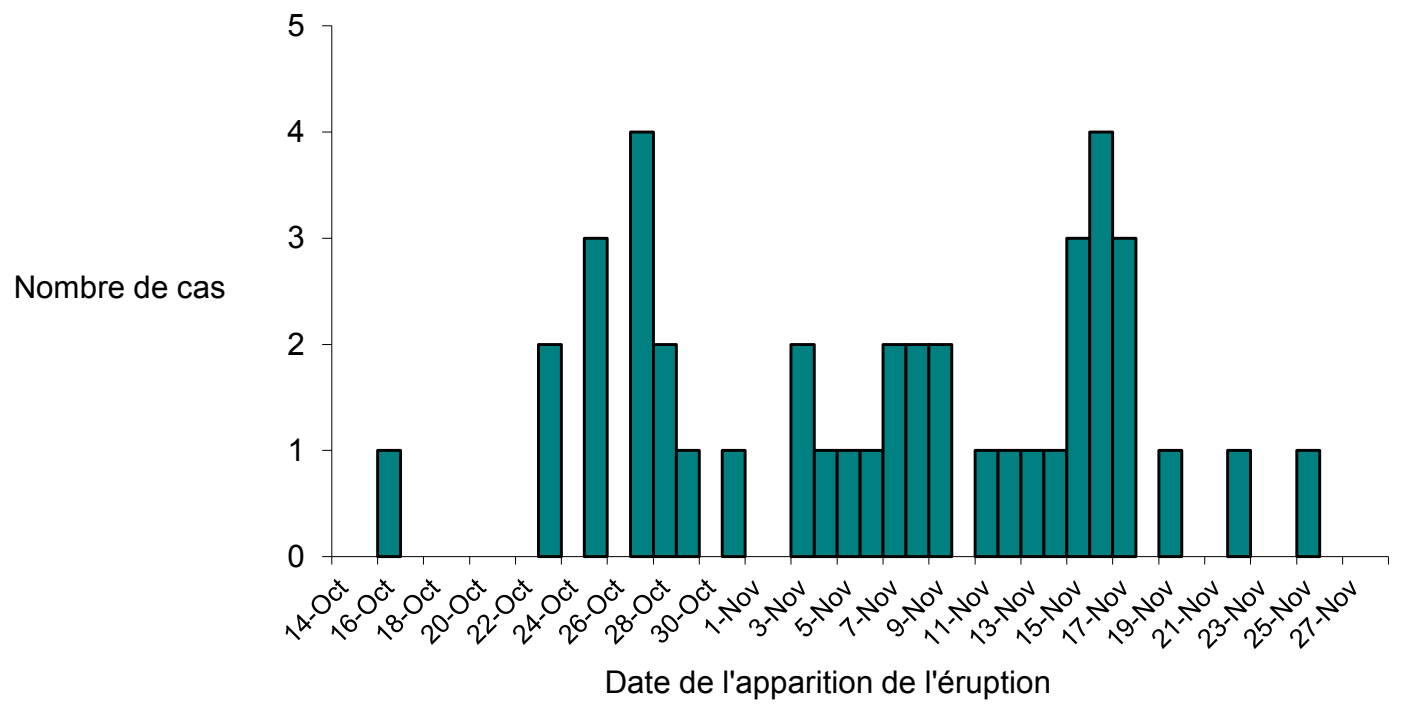

\section{Références}

(1) Stone SP, Cooper BS, Kibbler CC, Cookson BD, Roberts JA, Medley GF et al. The ORION statement: Guidelines for transparent reporting of outbreak reports and intervention studies of nosocomial infection. Lancet Infect Dis. 2007;4:282-8.

(2) Kershaw T, Suttorp V, Simmonds K, St. Jean T. Outbreak of measles in a non-immunizing population, Alberta 2013. CCDR. 2014;40:236-242. 